\title{
RECORDING HUMAN INTERACTION IN NATURAL SETTINGS
}

\author{
Charles Goodwin
}

This is a guide to the work practices, and video, sound and computer equipment, needed to record human interaction in natural settings. It is basically a description of how I work. Other people certainly have different ways of doing things, and these might be better than the ones I describe here. These are only meant as suggestions. However, in the interest of clarity I've stated things as directly as possible, without hedges and qualifiers. Moreover what is said here is largely confined to basic technical issues. At a few places I've included detailed cabling diagrams and descriptions of software procedures. Though these might not seem necessary for the casual reader, they are precisely the things that can take a long time to track down on your own. I therefore thought it would be helpful to include them.

\section{Video Formats}

- Hi-8 is better than VHS, $8 \mathrm{~mm}$ and probably Super VHS. Use it if at all possible (unless you have professional equipment). It produces much better copies than the other formats. You can shoot your original with $\mathrm{Hi}$ 8 and then copy into VHS for analysis. The copy will be a lot better than if you had used VHS for your original. Moreover Hi-8 tapes are much smaller than VHS tapes, which is a big help in the field. You can carry a couple in your pocket so that you are always ready to put a new tape in the camera when the old one runs out.

- Your analysis deck should

- Be able to play the same segment repetitively

- Have a counter that records in minutes and seconds instead of tape revolutions

- Despite a horrible early experience with Sony's first portable video (all of my original tapes disintegrated because of the chemicals Sony put in the backing) I use Sony equipment. One story I've heard and believe is true is that Sony and Apple are working together on future interfaces. Basically Apple would use the Sony remote control language to control video equipment from Macintosh computers. I believe (but of course am not certain) that Sony equipment made now will be able to be used this way through their current remote control ports. I don't know if Sony licenses its remote control language to other manufactures. Though I haven't used them I've seen Canon $\mathrm{Hi}-8^{\prime}$ 's which also look good. One Canon model allows you to entirely remove the lens and replace it with a different one, something that would enable you to do much better wide angle recording. 
- While Sony Hi-8 cameras are reasonably priced (and the best consumer cameras I have ever used) Hi-8 editing decks are very expensive (one theory holds that if Sony lowers the price on its editing equipment television stations will stop buying Sony's much more expensive professional equipment and switch to $\mathrm{Hi}-8$ ). I therefore record my originals in $\mathrm{Hi}-8$ and switch to VHS or $8 \mathrm{~mm}$ for analysis.

- The camera I currently prefer is the Sony V801. This writes a nonstandard time code on Hi-8 tape as it records. You don't have to record the time of day on the tape. During the playback you just hit the Time button and the time to the second at which it was recorded appears. The code itself can't be copied to working copies but you can copy the visible display (i.e. you can't turn the code on and off on your copies). You also get time into the tape in minutes and seconds that is accurate to the frame number. You can electronically edit using frame numbers with the Sony RM-E700 Time Code editing controller. The camera has Stereo sound (highly recommend - see below) and a ten to one zoom. The V801 has been replaced by a newer model, The EVO150TR which still writes time code, and has a color viewfinder. Highly recommend. A lot of Sony's latest models seem designed to be as small as possible. It might be that these new models are as sturdy etc. as the slightly larger ones, but somehow I trust the bigger cameras more.

Moreover these bigger cameras are still quite small and given that you will also be carrying a tripod and microphones, the slight difference in camera size of the smaller cameras has no real advantage. Before using the V801 I used the Sony V101 and found it to be the best video camera I'd ever used. Superb picture quality. The V801 is basically the V101 with a few new things added (time code, longer telephoto in the zoom).

\section{Hi-8 Videotape}

- Hi-8 technology is made possible as much by the tape as by the camera. You need special tape to record in $\mathrm{Hi}-8$. If you use ordinary $8 \mathrm{~mm}$ tape in a Hi-8 camera you will record in regular $8 \mathrm{~mm}$, not $\mathrm{Hi}-8$. Moreover you can't play Hi-8 tapes on regular $8 \mathrm{~mm}$ decks.

- There are two kinds of Hi- 8 tape

${ }^{\circ}$ HMP Metal Particle Tape. Pros prefer this because the tape itself (or the way in which the magnetic media are attached to the tape) is stronger. The tape can take a lot more abuse as will occur when it is used for editing with extensive jog and shuttle action which winds over the same section of the tape repeatedly.

${ }^{\circ}$ HME Evaporated Tape gives a sharper picture but is more fragile.

${ }^{\circ}$ In making a decision between these think of how you will be using the tape. If you are not making an edited film, but instead will work largely from copies of the original tape, going back to it only for 
segment tapes and new copies, you will not be putting the stress on it that a professional making a film would. On the other hand the marginal improvement in picture quality that you get with evaporated tape is probably not significant.

\section{VideoTaping}

- Practice with your equipment before you actually tape

${ }^{\circ}$ Get the camera instruction manual and go over it and the camera thoroughly, before you film.

- Set the time of day on the camera.

- Try out your whole setup first. Tape something at home. By actually working with the equipment you will discover problems that you hadn't imagined, and be able to fix them before you are in the situation where the recording counts.

\section{- Extension Cables}

- Take an extension cable and a multi-outlet plug to the field with you. If you have to use batteries take more than you think you will need, and practice inserting and removing them.

- Record the time of day on your tape.

- This provides an easy and precise way to locate any section of the tape when you do your analysis. Moreover this marking will always be the same, even on copies.

- Set the time counter before you get to the site where you want to do your shooting.

- If you are using more than one camera you can use the times on the tapes to locate the same moment on different tapes. Make sure that you synchronize the times on the cameras before you start. It is easy to get them so that there is far less than a second of difference.

\section{Sound Recording}

- Use an external microphone placed as closely as possible to the people you are filming. You will get much better sound this way. Test this yourself.

- If people are going to be in a limited area you can tape the microphones in positions near them before they arrive. Try the ceiling or lamps.

- Duct tape is invaluable. Always take it. It's great for taping microphone cables. If possible run them along the ceiling. If you must run them on the floor put a line of duct tape over them. That way people won't trip on the cables. duct tape comes in both silver and black. Black is less conspicuous. Companies that service professional film makers sell Gaffer's tape which is superior to duct tape. The glue is better and never leaves any residue. 
- Get extension cables for your microphones. I always carry three 25 foot XLR extension cables in my camera bag and use them all the time.

- Remove the batteries from you microphones when you are finished taping and put them in again just before you shoot. This is another important reason to use earphones-you'll find out if you have no batteries. You can't avoid this problem by leaving the batteries in the microphone. You might find yourself with dead batteries.

- Cameras that record in stereo are not essential but have advantages. If you are taping a complex situation (a family dinner, a work setting) you can put two microphones in two separate locations, for example at opposite ends of the table. In doing analysis you can choose to listen to only one track at a time. You will find that some talk which is almost inaudible on one track (i.e. it was far from that mic) can be heard clearly on the other.

- After some experimentation the Workplace Project found that TRAM TR50's give pretty good sound. Other microphones certainly will as well, but we were surprised at how much variation there was, and how much better sound we got with these. They cost about $\$ 200$. They are lavaliere mics but I rarely use them that way. Usually I duct tape one near where people are talking. I consider one of the microphones as essential as a camera and tripod.

- If you expect people to move from one place to another, you can save yourself a lot of setup time if you put mics in both locations initially (perhaps taped in place). Then all you have to do is move your camera and plug in the new mic.

- If people are going to be moving, and you have to follow them around with your camera, a shotgun mic mounted on top of the camera gives much better sound than the camera's built-in mic. Better yet is using two people, one to handle the camera and the other the microphone. I originally used a shotgun that I got at the a discount consumer electronics store for about $\$ 100$, but after making tests found that the Sennheiser ME-80 gives significantly better sound. The Sennheiser is a modular system consisting of a basic power supply to which you attach different microphone "heads" with different sound characteristics. You buy each component separately. The K3U Power Supply costs about $\$ 200$, the ME-80 Short Shotgun about \$212, and the ME-40 Cardioid about $\$ 150$. The Cardioid is directional, but not as directional as a shotgun. It eliminates most of the sound to its rear while picking up a rather wide area in front of it. There are lots of situations where the ability to do this is very, very valuable. The ME-40 Cardioid is also an excellent mic to hang over a table to record a conversation. Foam windscreens substantially improve the quality of your sound. The ME-80 takes the MZW-415 (\$37) and the ME-40 the MZW-30A (\$19). Sennheiser 
also makes an omni-directional lavaliere head for this system which is highly recommended and long shotgun which is not recommended.

- Professional sound people strongly recommend not using a shotgun mic in a horizontal position pointing from the camera to the people you're recording. Pointed this way your microphone will pick up not only the group you're recording, but also all of the sounds behind and in front of them. Instead try to get the microphone over the group pointing down toward the ground. That way nothing but their talk is in the mic's field of view. In natural settings this can be both hard to do and intrusive, and in some settings pointing a shotgun mic at the group you're taping will work fine. However, when the mic can be positioned over the group there is a great improvement in sound quality. Look for ways to hang your mic over the place where a group will be talking, for example over a table.

- I haven't used wireless mics but would very much like to have good ones. It would help immensely. I've heard that cheap wireless mics have problems (drift in and out or fade as people turn away - this is all hearsay). I've also been somewhat concerned since situations like the airport have a lot of radios and other electrical equipment that I don't want to interfere with. That said I think that wireless mics would be very, very valuable. You could follow people who are moving, eliminate cable in fixed settings, and eliminate a lot of setup. Stringing mic cables is one of the most time consuming and intrusive components of setting up to shoot. Samson Stage II series wireless microphones, such as the MR-1, have been recommended to me. They cost between $\$ 300$ and $\$ 450$.

- Get to know whatever mics and recorders you use well. Some of them have settings, probably designed for loud rock shows, that lower the signal. You don't want that for taping in natural settings.

- Tram mics, and many other mics, come with 3 pin XLR adapter cables while most camcorders have mini-plug mic inputs. On the next page is a diagram showing you how to wire adapter cables. It includes a diagram for making a very useful cable that will allow you to attach two separate external mics to the stereo input jack on a camcorder that records in stereo.

- I have found that this cable works fine when two mics are attached. When only one mic is attached it has to be attached to the left input connector. If it is attached to the other one you won't get any sound. Experiment to find out which input works on your camera and then mark that connector with a label. You can also attach a single external mic with the single adapter cable diagrammed on the bottom of the page. You can also use a stereo instead of a mono mini plug on such a cable and send the signal from your mic to both the left and right channels of your tape. 
- Soldering is quite easy and it is very helpful for making audio cables. You want a comparatively low powered soldering gun or iron. A wire stripping tool, set of needle nosed pliers and small vise are very useful. Strip the insulation from around the wires, twist the wire strands together, and attach them to the connector you will be soldering them to. Don't rely on the solder alone to hold everything together. Instead try to have the wire itself act as a tie. For example if the connector has a small hole (as most do) force the wire through the hole and bend it into a $U$ shape and wrap it back over itself. With the soldering gun heat the wire not the solder. Hold the solder onto the wire away from where you are heating it. When the wire gets hot enough the solder will flow around and through it. To check your connections use a continuity checker. This can be as simple a flashlight light with a needle at one end and a probe at the other or you can use a volt/ohm meter. Basically you put a probe on say the tip of the plug you've soldered and touch all of the wires in turn with the other probe. The needle should move (or the light light up) only on the connection you want (for example tip and wire attached to number 2 pin on the XLR). If any other combinations light up you have a short circuit. 

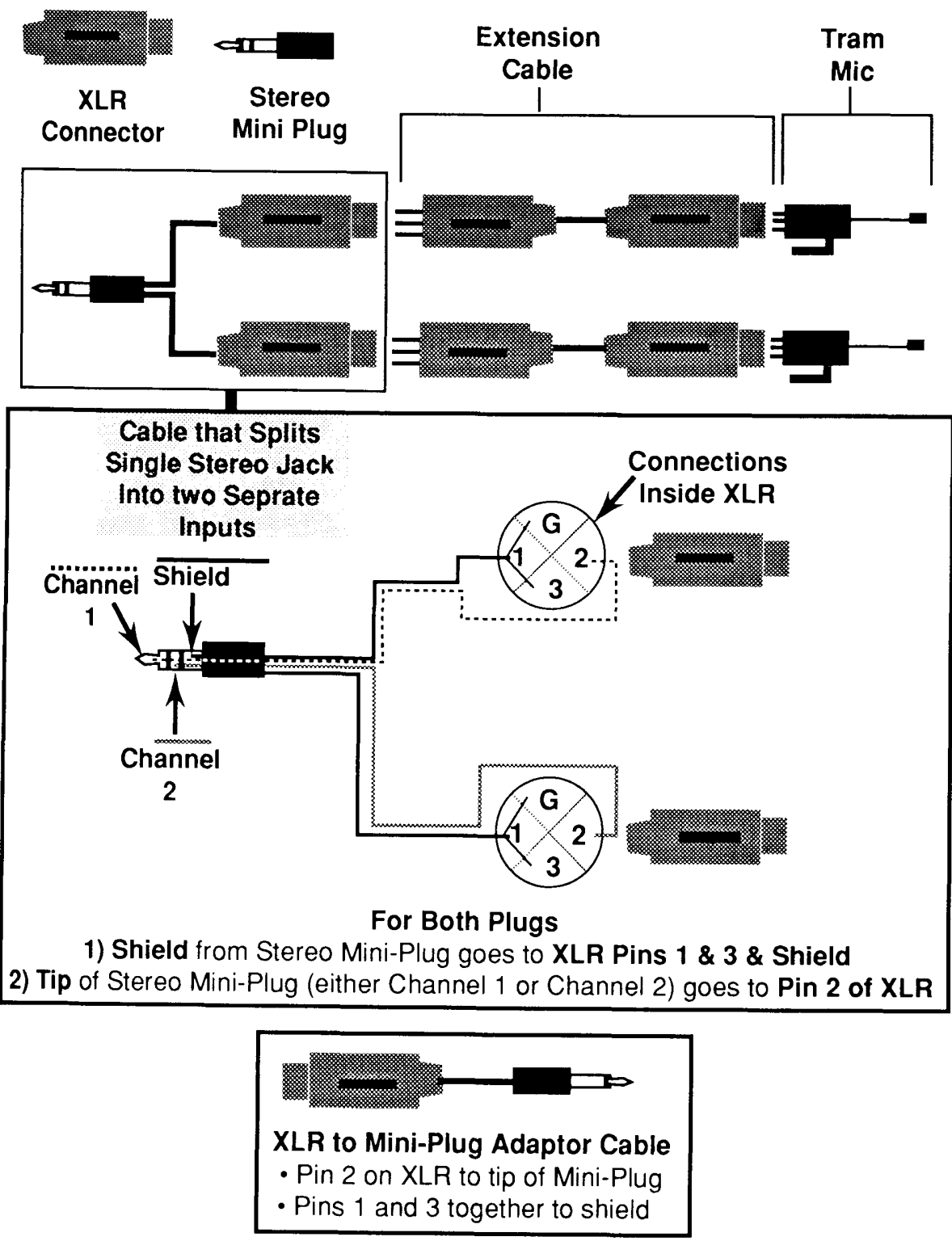
- The input jacks on consumer jacks are fragile. To prevent the weight of XLR adapters from straining them I wrap the cable above the adapter around something on the tripod head. Since the adapters are not hanging no stress is put on the mic input jack.

- Always use earphones.

- I taped some wonderful stuff and then when I got back home discovered that my microphone had broken.

\section{- Swiss Army Knife}

- A Swiss army knife is invaluable both in the field and when hooking up equipment for talks, etc. Make sure that the one you get has a Philips head screwdriver, a regular screwdriver and a scissors. I use the leather punch to remove batteries from the Tram mics, and the knife itself to remove plastic wrap from things like tape packages.

- The sound you hear in the field will never get any better when you get home. Get the absolute best you can in the field since everything will go downhill from there.

- Many people seem to think that somewhere a set of magic filters exist that will turn bad field sound into crystal clear audio. Some people also believe in Santa Claus. There is little, if anything, you can do to improve bad field sound. Instead concentrate on getting the best possible sound when you are actually recording. Move the mic as close as possible to the people you are taping. Try to get rid of as much background sound as possible. If the participants permit you, turn off air conditioners (bad white noise) and radios. Better yet try to pick field settings where problems like these don't exist in the first place (needless to say this can't always be done, and your main concern is the integrity of the setting you are working in). Mainly be very paranoid about the quality of sound you are hearing in the field. At the beginning of your setup do everything possible to maximize this. If appropriate make some changes later, but once activity in the setting gets going you probably want to avoid disrupting things by running in to move microphones (though there are circumstances where this can be done).

\section{Lighting}

- It would be great to be able to set up really good lighting. However extra lights are one of the most intrusive things you can add to a natural situation. I never use a movie light on top of the camera. I used to sometimes replace the regular lights in a room with 200 watt bulbs (I also carried spare fuses). This is no longer really necessary. Modern cameras do well in rather low light. Nevertheless try to get as much light as you can. 
- More important than the amount of light is its direction. If the area behind the people is much brighter than the people themselves (for example people sitting in front of an open sky or a white wall) this backlighting will make the people themselves appear very dim. Avoid this if at all possible. Don't shoot with a bright open window behind the scene. Some modern cameras do a good job of compensating for this, and have controls to manually override the camera's default settings.

However I've never found this completely satisfactory. The best thing is to avoid such situations.

- Similarly harsh sun can create dark shadows. If shooting outdoors I like to shoot people sitting in the shade (Before shooting Auto Discussion I had someone help move the picnic table under a tree. The tree also provided a place to hang the microphone).

- Lighting inside homes is frequently bad for taping. Try to get a ceiling light rather than lamps, especially lamps you are shooting toward.

- Fluorescent lights usually work pretty well (despite what you might have heard about their color balance they usually give a lot of light and people are easy to see).

\section{Tape Considerations}

- Carry backups of as much equipment as you can, e.g. an extra microphone, extra batteries, etc.

- Label your tapes as you are shooting.

- Put a label on the spine of the tape.

'Write the setting, date and tape number, e.g. Archaeology Dig 22-Jul92 \#1.

${ }^{\circ}$ Leave room at the top of the label to write your final catalogue number later (i.e. you want to have all your tapes in order. The number you put on at the site simply indicates if this is the first, second, or third tape shot that day).

'Don't worry about labeling the tape boxes. It's more of a nuisance than a help in the midst of actual setting (the easiest thing to do is to put the tape you remove into the box from the new tape -- therefore you don't want the boxes labeled).

- If you use more than one camera have a letter in your label for each camera. Make notes where each camera is positioned. That way if you later want to find the tape that was focused on the left side of the room, and your notes tell you it was the B camera you just have to look for a tape with B in the place where you mark the camera on the label. 
- Always hit the record tab on a tape that has been shot as you remove it. That way you can't accidentally record over it. This also provides a quick way to tell if a tape has been used yet.

${ }^{\circ}$ On $8 \mathrm{~mm}$ and $\mathrm{Hi}-8$ tape there is a sliding red tab that you move. On VHS tapes you break a little plastic tab on the spine of cassette. If you later want to record over the tape you can put a piece of scotch tape over the hole created when you broke the tab.

- If possible make notes as you are shooting.

' Use a small pocket notebook. Anything else is too big to handle in the midst of everything else you are doing.

- Notes are especially important on a multi-day field expedition. At least indicate the date when a tape was shot and roughly what was happening.

- If you record the time of day on the tape you can note specific incidents even as they are happening. Just look at your watch to get the time and make a note in your notebook. Later you can find that place on the tape.

- Shoot continuously. After all of the work and effort you put into getting to the site where you want to film, and the cost of equipment, the cost of tape is peanuts. You want to get everything you can. Bring plenty of tape, certainly more than you think you will need. You can't anticipate when interesting things will happen and if you record everything you can later go back and see what led up to them.

- If possible note the time that you start a new tape. Then you can look at your watch to figure out when the tape has to be changed. (I don't always manage to do this -- sometimes things are too hectic. But I'm glad when I do do it).

\section{- Use two hour tapes.}

- If possible try to avoid recording on the first two minutes and last two minutes of the tape. The signal is much more apt to jump, etc there. I usually don't do this because there is too much going on when trying to tape natural interaction. However I do notice some jumping at the beginning and end of a tape.

- Whenever possible (e.g. at least $95 \%$ of the time) use a tripod. It is much, much easier to look repetitively (as you must doing analysis) at a tape that is steady. On some occasions a tripod is impossible (following someone around for example). Practice holding the camera as steady as possible. Don't rely on just one hand. Put your left hand under the camera to support it and try to move as smoothly as possible.

- Bogen Magic Arms are invaluable when taping in natural settings. You can clamp the arm to a door, window, chair, etc. and then easily position 
the camera anywhere you want it. A ball and socket head on top of the arm to fine tune the camera view is invaluable. The Bogen Magic Arm comes in two parts, the Magic Arm itself (Bogen 2929 or 2930) and the Super Clamp (Bogen 2910). The Bogen 2929 is easier to adjust and lock than the Bogen 2930, but both will work. I use a Gitzo 175 Ball and Socket head. It is medium to small sized which I find adequate for a light $8 \mathrm{~mm}$ or Hi-8 camera. In order to get the camera and ball and socket on and off the arm easily I use a Bogen Quick Release Plate and Mounting bracket. When using the Magic Arm it is an immense help. The arrangement of Magic Arm, Quick Release Plate and Ball and Socket Head I highly recommend. I also use the Quick Release Plate on my regular tripod. I like it very much but could live without it if I had to (which I wouldn't say about the Quick Release on the Magic Arm).

- Position the camera to get the best view of what you are trying to tape, not where it is most comfortable for you. Sometimes the camera has to be quite low. On the newer $8 \mathrm{~mm}$ and $\mathrm{Hi}-8$ cameras you can easily tilt the viewfinder up so you can look down at it instead of having to get behind it. Other times you might have to put the camera quite high, for example on top of a file cabinet or attached to a Bogen arm. Such a position might give you a much better view of the materials that people are working with than a waist level shot would.

' On many occasions the best place to put the camera is somewhere where you can't get behind it (e.g. on a file cabinet, in the corner of a dinning room). A small, handheld monitor is invaluable in such circumstances. You can hook it up to the camera while positioning it in a place that is comfortable for you and reach up and adjust the camera.

\section{Wide Angle Lens}

- Just about everywhere I've taped I found a wide angle lens to be very important. On most modern video cameras the lens can't be removed from the camera. This creates some problems. You can't get a really wide angle lens and the picture quality is somewhat degraded when you attach one. Despite this you really need it. However, there are many times when you don't want to use it. If you can get what you need without using it do so. If you want to get a close-up of what people are writing you want it off the camera. Very frequently I attach and remove wide angle lens several times during the course of a day's (or even an hour's) shooting, especially if the events I'm taping are changing (for example workers at an archaeological site).

\section{Multiple Cameras}

- Multiple cameras are extremely useful in many situations. That way you can get both a wide angle that covers all participants and close-ups of 
specific events. It is also possible to cover a complicated setting that couldn't be covered with a single camera. In the setting reported in Transparent Vision we used three cameras, one on each side of the room, and one roving close-up camera. For the analysis developed in that paper all three were necessary. Some of the interaction was visible on only one or the other camera, and the close-up provided us with a shot of what everyone was looking at, which couldn't be clearly seen in the wide angle cameras.

- You can get electrical equipment that will feed several cameras into a single image, for example a different camera in each corner of the screen. When you do this the recorded image from each camera gets a lot smaller. If you are going to do this have each camera make its own tape before the signal is sent to the mixer.

- Professional cameras write time codes that can be used to synchronize them.

- What I do, which is not ideal, but works for me, is simply use the time of day clocks on each camera to visually locate equivalent places in the tape recorded by each camera. Thus if something I'm interested in happened at 7:02:34pm on the A camera I look for the same time on the B and $C$ cameras. As noted above you should synchronize the clocks so that they have less than a second of difference between them before you start taping.

- You can also use this to set up multiple views of a screen on separate monitors placed next to each other. However it is cumbersome.

Moreover you can't just start separate tapes together and expect them to stay together for a long time. Each recorder plays at a slightly different speed and eventually the tapes drift out of alignment. If you want to really keep synch you need time code.

- I haven't tried this but a computer program designed to edit movies on the computer itself, such as Adobe Premiere might enable you to bring in clips and sound from separate cameras, line them up and look at them in relationship to each other.

\section{Camera Bag}

- This sounds minor, and is certainly not as important as having a good camera, microphone and tripod. It is however more important than you might think. You want to get into a setting and set up with as little fuss as possible. This is much, much easier if you have all of your equipment in just one or two bags. My bag holds a camera, several videotapes, two lavaliere mics, one shotgun mic, one other mic, three $25^{\prime}$ microphone cables, a long electrical extension cord with a multiple outlet strip, a tiny monitor with its power supply, the camera power supply, rechargeable batteries for the camera, AA and button cell batteries for the 
microphones, monitor, etc., a bunch of adapter cables, a pen, some duct tape, the manual for the camera, etc. It is a tremendous help to have all of this stuff in one place. In that I keep it packed I have whatever I need whenever I go tape. Make sure that your camera, and any other delicate equipment is well cushioned. The bag I use is a Tamrac model 613.

\section{Recharging Batteries}

- The rechargeable batters that Sony sells for its $8 \mathrm{~mm}$ and Hi-8 cameras have a memory. If you recharge them before they are completely discharged they will start to go empty after only a little while. You won't get much recording time out of your batteries. To avoid this always discharge your batteries completely before recharging them. Don't top them off after using them for a short time.

${ }^{\circ}$ I discharge my batteries completely after every day's taping. To do this I attach them to a camera and play a tape. I leave them discharged until the night before I tape again. Then I charge all the batteries I will take.

- You can also buy a special gadget, such that the Sigma Powermax Discharger, that is designed specifically to discharge your batteries. I just purchased one and was surprised at how much life I got out of my batteries after using it.

- Sony's latest power supply/charger automatically discharges batteries before recharging. Older models don't.

\section{Still Camera}

- Recently I've also taken a still camera with me when I shoot. While this can be very, very useful, and is a great aid for ethnographic documentation, I find that $99 \%$ of my subsequent analysis is done with the video. The stills are less important now that frame grabs are possible. They are nonetheless invaluable for certain things. A camera that prints the date or time on the image itself is especially valuable. You can get backs that do this for professional cameras like Nikons, and some of the smaller point and shoot cameras for consumers also have models that do this.

\section{What to include in the Frame}

- This is the most important topic in the handout. However it is not one that can be answered in a simple way. Any decision you make will have problems. For example if you shoot wide angle to get all the participants you won't have good close-up views of their facial expressions or the materials they are working with. If you shoot close ups you'll miss the interaction (something you never want to do). Basically your analytical interests should govern your choice of what to include, and indeed the 
decisions you make here are in and of themselves a central part of your analysis. Moreover they will shape and constrain whatever you are able to do with the tape subsequently.

- Since I have been interested in interaction, and very much in what hearers as well as speakers do, I have always included the recipients in the frame. This does however mean zooming and changing camera position in order to get the relevant participants as large as possible, and sometimes deciding that someone is not a relevant participant at the moment (clearly a contestable analytic decision). You can see some of this in Auto Discussion. When working on the oceanographic ship my lens would sometimes not cover the entire room so I had to make decisions about which group to cover. I also wanted a record of the computer screens they were working with, and sometimes I zoomed in on them. Though I lost the participants briefly these shots were invaluable for subsequent analysis.

${ }^{\circ}$ I view taping as an iterative, progressive process. The first time you tape in a setting, and then work with the materials you've collected, you find both wonderful things and problems in what you've done. What I like to do at this point is go back and try to get the stuff I missed the first time. For example, when working the airport settings at the Workplace Project we quickly realized that the materials that participants were working with, such as documents and computer screens, were crucial to what was going on. We went back again and again trying to find ways to capture the work practices, the interaction, and the documents. This involved working with multiple cameras and experimenting with different ways to place the camera. It's not that some of the taping we did we was right and some was wrong, but instead that the analysis we were engaged in and the process of collecting data were both parts of a single recursive process. This was just as true for my earliest work in taping conversations. The first time I taped I hand held the camera and got sea sick looking at the tape. I quickly realized that I needed not only a tripod but also a wide angle lens when I tried to tape in people's dining rooms and kitchens. Then I discovered that I had to be able to move a lot of equipment quickly so I built a cart to carry everything (this was invaluable for Clacia and Auto Discussion). In some of my recent work however I've found it necessary to hand hold the camera.

- Some of what you bring to a setting is a way of seeing developed in earlier settings. For example when I first taped archaeologists I got interested in the way that they would map artifacts and moved the camera every which way to try and capture the different components of this process, which included at least two and usually three 
separate people spread out in space, tools such as rulers and plumb bobs, and writing on different kinds of surfaces. When I encountered this situation again several months later I knew right where I wanted to be and was able to move the camera there immediately and get some very useful data.

- A major question: How to integrate ethnography with taping? No simple answers here.

\section{Tape Library}

- Have a sequence of consecutive numbers for all of the tapes in a particular project. That way you both find a tape easily, and tell immediately if one is missing. At one point we used a date code to label tapes (e.g. 91-10-03 \#1). While this gave each tape a unique number you couldn't tell if one was missing. WV-01, WV-02, etc. is simpler and clearer.

- Keep a tape log.

- The best place to do this of course is on the computer. I adapted my address book program, Dynodex, to do this.

- I use separate number codes for originals from each project (e.g. CRT-19 is a tape of Courtroom Data, ARL-13 was taped in an Archaeology Lab). The codes are short so that they can be written, along with the date, on labels pasted on the tapes. I then have a code for my working copies (VDE for $8 \mathrm{~mm}$, i.e. Video Data Eight, and VDV for VHS working copies).

- I color code all of my tapes (I'm really paranoid about messing up my originals). I got small dots from a stationary store and put red dots on originals, green dots on working copies, and yellow dots on segment tapes. This way I can tell at a glance what kind of tape I am working with.

- In the entry for each original in the tape log I mark the tape number of any copies that have been made. When I want to find a particular tape I look under the originals and the log immediately tells me the tape number of the copy I need. When working with data, even though I am working from copies, I always use the code for the original to note where a particular piece of data came from.

- If possible keep your originals in an air conditioned room that few people have access to. Copies should be easy to get to.

- You might want to use your computer to make your own tape labels for $8 \mathrm{~mm}$ and Hi- 8 tapes. A piece of paper can be folded in the $8 \mathrm{~mm}$ plastic boxes to make a label. This provides an easy way to put your name, address, Tape \# and comments with the tape. I adapted the audio tape 
labeler that came with Hypercard, but you can probably do the same thing with a word processor.

\section{Copying Tapes}

- ALWAYS work from copies not originals.

- Even if you think you want to focus on a particular segment copy tapes from the beginning right through to the end. Don't go to just the segment you want. This way the counter numbers that you are working with on the copy match the counter numbers on the original. Thus if your data segment is 33 minutes into tape ARL-22, all you have to write is ARL-22 0:33 and you can immediately find it both on any copies and on the original. Otherwise you spend a lot of time searching through the tape any time you go back to the original to make a segment tape or frame grab.

\section{Sound recording during copying}

- Most modern consumer video cameras set sound levels automatically. However some decks you will use for analysis, as well as good audio recorders, allow you (or force you) to set the record level manually.

- To do this you use a meter. Sometimes it is a real meter, sometimes flashing lights. Irrespective of these differences what you want to do is

- Play through a bit of the original tape until you get what you think will be the loudest sounds that you'll copy.

- Set the record level on the recording machine so that these sounds just peak over the optimal setting (marked on the meter or the lights -your manual will tell you.)

"Leave it at this setting. Don't try to "ride the gain" by constantly adjusting the level as the recording is made. You will only make things worse (e.g. you'll raise the level when listening to low passages so that when a loud passage suddenly comes it will be way too high and get distorted).

- Cable Setup:

${ }^{\circ}$ Go into Line In (not mic) on the record deck. On some machines this is called $A u x$.

' Come out from the playback deck at Line Out. Only if there is no Line Out use the earphone plug. You get better sound from Line Out. Moreover you have to adjust the volume if you use the earphone. If you do have to use this set the earphone control at a mid level. Don't set it too high. Then make your final adjustments with record level control on the record deck (or let it get set manually).

- Incidentally a very good audio recorder is the Sony Professional Walkman WM-6DC. If you get it also buy a mic extension cable from 
Sony parts (outrageously priced). It gives you much better sound (you eliminate noise from the machine itself) and provides for better mic placement.

How to copy tapes (simplest system).

- You need two machines which I'll call the playback machine and the record machine. The tape you are copying from is on the playback machine and the new tape that you are making is on the record machine. The playback machine might be the Hi-8 camera you used to record the original, and the playback machine might be a VHS deck. The two machines do not have to be the same format.

- You need one cable for video and one for audio (two if you have stereo) to connect the two machines to each other.

- The video cable goes from Video Out on the Playback machine to Video In on the Record machine.

- Similarly the audio cable goes from Audio Out on the playback machine to Audio In on the Record machine.

- The output of the record machine is then hooked up to a monitor.

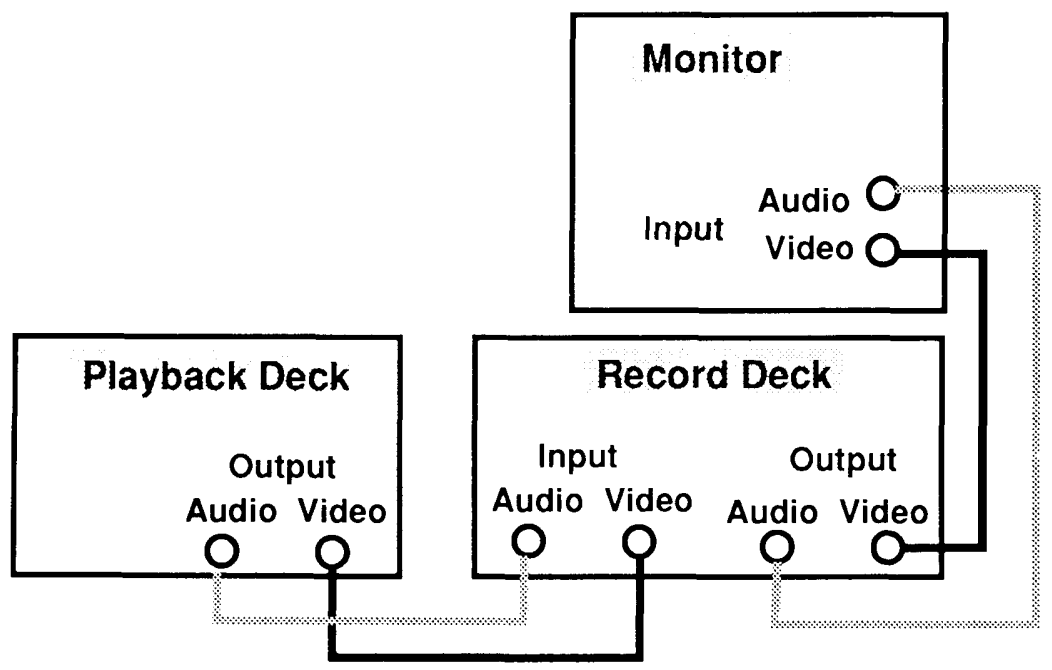

\section{Turn Edit Switch On on Sony Recorders}

- The Edit Switch on Sony VCRs controls special filtering. Turning the Edit Switch ON removes these filters from the circuit so that the recorder 
making the copying can do its own filtering. This produces a better copy.

- If you are using Sony machines put the edit switch ON on both machines when copying.

- Turn the Edit Switch OFF when not copying. This is especially important when using a camera to record new data. If the Edit Switch is ON you will get a much poorer recording.

- Make sure that the record tab is fixed on the original so that you can't accidentally record over it.

- Give the copy a new number in your tape catalog, make a label with this number and Copy XYZ-10, and put it on the tape that will be used for the copy.

- Put the appropriate tape in each machine (original in the playback machine and a blank tape in the record machine).

- Simultaneously hit the playback button on the playback machine and the record button(s) on the record machine.

- How to make a segment tape.

- When you give a presentation you want to put the brief excerpts you will show in order on a presentation tape. I call this a segment tape since it contains a collection of short data segments. You would also use this if you were making a collection of a particular kind of phenomenon.

- From your working copies get the data citations (e.g. tape number and location on the tape) of the segments you want, and arrange them in order.

- As you make a segment tape it is very useful to simultaneously write a table of contents or log. This will contain

- The counter number on the segment tape where each segment begins.

- The tape number of the original tape that the segment is taken from.

- The counter number on that tape where it was taken from.

- The time of day on the original where the segment begins and the time of day where it ends (this is really useful when actually making the segments).

- A brief description of the data segment.

For example: 


\begin{tabular}{|c|c|c|c|c|}
\hline Counter & $\begin{array}{l}\text { Original } \\
\text { Tape }\end{array}$ & $\begin{array}{l}\text { Org. } \\
\text { Counter }\end{array}$ & $\begin{array}{l}\text { In and Out } \\
\text { Times }\end{array}$ & Description \\
\hline $0: 00: 51$ & $\begin{array}{l}\text { ARL-21 } \\
\text { WARP \#4 }\end{array}$ & & $\begin{array}{l}1: 39: 00- \\
1: 39: 33\end{array}$ & Go to 90 Sequence \\
\hline $0: 1: 25$ & $\begin{array}{l}\text { ARL-18 } \\
\text { WARP \#1 }\end{array}$ & $1: 45$ & $9: 30: 48$ & Munsell Sequence \\
\hline $0: 02: 13$ & CRT-20 & $17: 45$ & $\begin{array}{l}0: 18: 12- \\
0: 18: 30\end{array}$ & King Being Beaten \\
\hline $0: 02: 36$ & CRT-19 & $15: 35$ & & Sergeant Duke \\
\hline
\end{tabular}

- With a log like this you can both easily find the segment you need when making a presentation, and note exactly where each segment came from on its original tape.

- Machines are set up exactly the same way as for copying.

- Use your originals, not your working copies, to make a segment tape. Tape quality deteriorates rapidly when copies are made from copies.

- Record perhaps 30 seconds of blank at the beginning of the segment tape (the tape in the record machine)

- Put that recorder in Pause

- Start the original a bit before the beginning of the segment you want to record (to allow the tape to come up to speed).

- When the tape reaches the point where you want it to begin Release the pause Button on the record machine.

${ }^{\circ}$ Here is one place where time of day on the original tape is very helpful. After you've determined your segment boundaries just look for those numbers. It's a lot easier than trying to remember word cues, etc.

- When you reach the end of the segment turn off the playback machine (but not the Record machine).

- Record a couple of seconds of blank as a division between segments.

- If you can get to your next segment very quickly put the Record machine in Pause while you set up the next segment on the playback machine. If it's going to take you a while turn the record machine off (it's not good for tape to be held in pause too long, and if you wait too long the recorder will start again automatically).

- This method avoids the problems of trying to get clean cuts with insert editing. While this kind of editing, and recording a tape that already has black recorded on it, is preferable and necessary for an edited tape, it is 
not necessary when all that is being done is stringing a series of segments together.

\section{Tape Citations}

- Whenever you find a bit of data you want to work with you want to know exactly where it came from. Ideally your citation would have three pieces of information

\section{Tape Number}

ARL-30

\section{Time into Tape} $0: 50$
Time of Day 2:52pm

- In practice you might not always have the time of day. The time into the tape is very, very useful. If you have it you can easily find that segment on the original or another copy (as long as you have copied the whole tape from the beginning). It also makes it easy to search for that and other data on the tape.

- Clearly playback decks with counters that record in Hours, Minutes and Seconds are much, much more useful than those that simply record tape revolutions. These aren't even constant throughout the tape (e.g. the amount of tape spooled in a revolution constantly changes as the diameter of tape on the feed and takeup hubs changes).

\section{Content Logs}

- It is very useful to have a rough log of what any tape that you shoot contains. View this as a rough guide to the tape, a way to quickly find things in it, rather than as any kind of definitive description of what is on the tape.

- As your analysis changes, for example as you discover new phenomena, things that you might initially have passed over on the tape suddenly become very interesting. "What's on the tape" emerges as process of interaction between the tape itself and the interpretative framework you bring to it, something that it is a continuous state of flux. Thus, no content log is ever definitive. However, don't let this prevent you from making any content log at all.

- In a content log include the Time into the Tape, the Time of Day (from the on screen counter) and your own rough notes. The files containing all of our Content Logs start with CLV (for Content Log Video). That way we can immediately tell what kind of file it is. 


$\begin{array}{ll}6: 00 \mathrm{pm} & 2: 06 \\ & \\ 6: 25 \mathrm{pm} & 2: 06: 30 \\ 7: 15 \mathrm{pm} & 2: 07 \\ 7: 30 \mathrm{pm} & 2: 07\end{array}$

$8: 30 \mathrm{pm} \quad 2: 08$

9:15pm 2:09

9:57pm 2:10

CLV ARL-1 Arch Lab 16-Sep-91

Ann tells Nancy what choices are for a particular category

Betty comes in, introductions of everyone in lab Discussion as to whether something is a label Tell them to go to notebooks -- way that construct narrative "it was raining etc." to make sense out of the materials as they now present themselves Looking at coordinates on chart on bookcase Tells Nancy to look for Rims -- now that have been washed. Directive Chuck's coke explodes After goes out of room reconstruct what sound meant to them -- i.e. "at first I thought" stuff

- I find that the best time to make a content log is when I'm making my working copy of the tape. That way I'm forced to get through the whole tape. If I do it with a copy I quickly get intrigued with something, or want to make sure that I'm right about what I think I see, and then start looking in detail at the segment that caught my attention. This leads to new analysis, but I never get through the whole tape. If I make the log while the copy is being made I can't stop it, but can still flag the things I want to look at in more detail.

- It is of course easy to make additions to the Content Log when you view the tape again.

- To find things later it is useful to use standard terms, "keywords" for phenomena.

- To make the log I use the Table feature in Word 5. You can add things indefinitely in the notes column and without interfering with the time columns. I keep a glossary entry for Content Log Columns on my Work menu so that it is available whenever I need it.

\section{Transcripts}

- I will not here discuss all the issues involved in transcription as theory, alternative representations, etc. These are descriptions of a few ways of doing transcript that I have found useful.

- I find the Table feature in Word useful for Transcripts (I actually use several different formats for transcripts I am using for different purposes). I add a Notes column to the right of the Time, Speaker and Utterance columns. 


\begin{tabular}{|c|c|c|c|}
\hline Time & Speaker & Talk & Notes \\
\hline $0: 13$ & Geoffrey: & $\begin{array}{l}\text { Not mu:ch } \\
\text { going [on. }\end{array}$ & \\
\hline & Pam: & scale. $\quad$ Hey: you're out of & $\begin{array}{l}\text { Unusual voice quality that } \\
\text { don't know how to capture } \\
\text { Note how like monitoring } \\
\text { in the Ops Room }\end{array}$ \\
\hline & & (6.2) & $\begin{array}{l}\text { Before answering Pam Pat } \\
\text { moves her gaze from Chart } \\
\text { to CTD } \\
\text { Getting information } \\
\text { relevant to production of } \\
\text { that answer. }\end{array}$ \\
\hline & Pat: & $\begin{array}{l}\text { No: } \\
\text { You can actually change the } \\
\text { (breadth). }\end{array}$ & $\begin{array}{l}\text { Said after look to CTD } \\
\text { screen }\end{array}$ \\
\hline
\end{tabular}

${ }^{\circ}$ Lines have been added to the printout to highlight the column arrangement. You don't need them in your printouts.

- I can add very long comments in the Notes column and the Transcript Lines will expand to accommodate them. If I want just the Transcript I can eliminate the Notes Column, and the empty space between lines of talk will disappear.

${ }^{\circ}$ This transcript format is for my own work. When I use it I have the paper print sideways in Landscape mode so that each row can be longer than normal.

- For papers I define a style in Word with tab stops for right justified line numbers, arrows, speakers and talk:

CRT-018 0:56

9 Defense: And what Sergeant Koon will tell you=

$10 \rightarrow \quad$ this is his [rendition, (0.4) of what he sa:w.

11 Smith: lOh No.

- To line up overlap simply put a tab before the overlapped talk, add a new tab to the ruler line, and move it until things roughly line up.

- If you want to line up tabs for overlap more precisely open

Preferences in Word and change the measurement unit from inches to points.

- It's a good idea to put overlap brackets on both lines of overlapping talk. 
- I find it very useful to add sub and superscripted brackets to my Work Menu so that I can insert them when needed instead of having to format each new one by hand. I put formats for content logs and transcript tables there as well.

\section{Using Video in Talks}

\section{Overheads}

- Use at least 18 point type on your overheads. If you use smaller type people will have a hard time reading it.

- Number your overheads in sequence in a small size (I use 10 point) on the bottom of the Overhead. If they get shuffled out of order you can immediately put them in sequence again.

- Putting multiple overheads on top of each other to develop an argument.

${ }^{\circ}$ Do one diagram that contains everything.

- Then print out subparts of that for each separate overhead.

- Since all the parts fit together in the master all you have to do is line up the edge of each overhead with the one before it and the images will be in exactly the right place.

- To make the separate frames from the master you can make a bunch of new files, starting with the master and then deleting everything you don't need for that particular overhead.

${ }^{\circ}$ With Illustrator you do the same thing with a single file

* Put a label for each layer outside the printing area.

* Group the label and whatever will be printed on that overhead together. Include a sequence number on the bottom right (e.g. AAA-03a, AAA-03b, AAA-03c, etc. Each small letter marks a layer in AAA-03) so that you can easily put things in order. Pile the sequence numbers on top of each other. Since each will be grouped with a label only one will print at a time). Now, just by hitting the label you can select the layer you want.

* To print each layer

- Select All (e.g. everything on the document)

${ }^{\circ}$ Use the Shift key to De-Select the layer you want to print.

${ }^{\circ}$ Choose Hide from the Arrange menu. Only the layer you want to print (the one that wasn't selected) is left.

${ }^{\circ}$ Print this.

- Pick Show All from the Arrange Menu to get everything back and proceed to the next layer. 
- I gather that Aldus Freehand can do this in a simpler fashion.

- You can print overheads directly on a Laserwriter. However you have to open the tray at the back of the printer so that the overhead does not bend around and come out on top. If you only have a single overhead you can put it in the external page feeder. If you have many overheads put them directly in the paper tray. Remember to remove them when you are done.

\section{Segment Tape}

- Have the examples you will need in order on a Segment Tape. Have a $\log$ that shows you the counter number where each segment begins. Since I use an $8 \mathrm{~mm}$ deck my Segment Tapes are $8 \mathrm{~mm}$.

- Have an Overhead with a transcript of what is said during the sequence. Text on the overhead should be at least 18 point.

\section{$V C R$}

- Bring you own VCR. If you've spent a lot of time preparing a talk you want it to work. I find that the VCR's that hotels and convention centers provide are terrible. Most of the time they do not allow you to replay a small sequence repetitively. If they have a remote control it works differently from your own so that your hand doesn't know what to do. The result of all this is a mess during your talk as you try to get the tape to play the segment you want. People want to hear your analysis, not your apologies for why you can't present it.

- If you bring your own VCR you know exactly what it will do and the remote control is second nature to you. The machine I use, and strongly recommend for this purpose is the Sony EV-C3. It is a small $8 \mathrm{~mm}$ deck, about the size of a dictionary, that you can easily carry on an airplane and it has a small but very adequate remote control. The deck does not have stereo but that is its only drawback. The counter counts in minutes and seconds. Sony makes smaller, pocket sized Watchman. However these don't hold a still frame well. When I first got the EV-C3 I liked it so much that I used it for all of my analysis. However after a couple of months of very heavy use I had to have it overhauled. It's not designed for a really heavy pounding but I still use it for a lot of my daily work and teaching.

- Take your own cables and adapters. Let the hotel supply the monitor. Different monitors have different inputs. Take a pair of RCA cables and then go to Radio Shack and get every conceivable adapter, including RCA to Miniplug and RCA to BNC. Also take a coaxial cable in case they don't have a video input.

- Hand carry your tape and have it inspected by hand when you go through airport security. Record stores carry small audiotape pouches 
that work very well for $8 \mathrm{~mm}$ tapes (the larger tape boxes that have a compartment for each tape DON'T work since $8 \mathrm{~mm}$ tape boxes are wider than audio tape boxes).

- I've taken this machine to Europe on many occasions and have always been able to get it to play there. Frequently you can find a multi-format monitor that will play NTSC, the American Standard. Even if you can't find one American tapes will sometimes still play. For example on English monitors you get a cinemascope effect with blank lines at the top and bottom but your tape playing fine in the middle. Carry a voltage transformer (you'll destroy your machine if you try to plug 220 into it) not just an adapter plug. The biggest problem I've had in Europe is that some monitors have input jacks which are totally unknown here.

- Use a cheap camera bag with padding to protect the deck as you carry it.

- Always get to your talk at least an hour before hand to set up you equipment.

\section{Work Setup}

- Since what you want to do as you look at tape is make notes, do analysis and transcribe, I find it essential to have the analysis deck and monitor right next to my computer.

- At this distance a 13" Video monitor is better than larger monitors. It is hard to find a monitor this size with an earphone jack.

- At the moment I think that Macintosh is the preferred computer system for doing either audio or video analysis (The Mac IIci is an excellent choice). With the Macrecorder you bring sound right into the Mac (this is now how I do transcription) and frame grabbing cards make it possible for you to capture images from the tape. Gene Lerner has written The Workbench for audio and video analysis and collection making. It requires the Mac. Adobe Photoshop is the program of choice for working with frame grabs. I also use Adobe Illustrator to add text and arrows to the image, and to make transcripts that incorporate both frame grabs and graphic annotations. I gather that Aldus Freehand would do just as well. I used to use SuperPaint but find Illustrator vastly superior. All of my overheads for talks are made with Illustrator. (Something that took me a while to figure out in Illustrator: To set up Tabs for transcript etc. you first draw a text box and then use the Pen Tool to draw a vertical line from the top of the box to the bottom where you want the new column to begin. Make this invisible by assigning 0 fill and stroke and then choose Make Text Wrap from the Type Menu. When you hit a tab text will start at the line. Basically you do tabs by acting as though you were trying to wrap text around an object, the line that you drew. Not at all intuitive and hardly mentioned in Illustrator's manual). Pagemaker allows you to incorporate both text and graphics on a page. However I've 
never really gotten into using it. I find that if I'm working with a single page Illustrator gives me a lot of control, more I think than Pagemaker would. When I want to write paper I use Word 5. I don't think that Pagemaker does footnotes automatically and it certainly doesn't support Endnote, a bibliography program I use all the time. Unlike earlier versions of Word, Word 5 allows you to incorporate graphics. The best way to do this is with System 7's Publish and Suscribe capabilities. However (and this took me months to figure out) this won't work unless you do the following. When you create a publishers in Illustrator or Photoshop select PICT rather than EPS in your options box. EPS publishers will look fine on the screen but will not print properly. You can also embed objects in the text with the Insert Picture command. The objects being inserted have to be in EPS format (a save option in Photoshop -- in Illustrator you have to choose Color Macintosh Preview). If you get lines across some of your graphics 1) Turn Fractional Widths $O N$ in Word; 2) In the Laserwriter Options in the Chooser turn

Background Printing Off. You won't be able to do anything else on your computer while the paper is being printed, but your graphics will print better. Turn Background Printing back on when you're done. Papers that include graphics take up an incredible amount of memory. Some of the papers I am working on end up over two megabytes when I've inserted a number of graphics. To work with Photoshop and Word 5 in this way you need a lot of memory, at least 8 megabytes of RAM. I now have 20 megabytes of RAM which allows me to have Word, Photoshop and Illustrator open at the same time. DiskDoubler is a good and safe file compression program, a necessity when working with frame grabs. For working with sound the program of choice is SoundEdit Pro. I use Dynodex, an address book, to keep my tape log.

- Frame Grabbing Cards: The card you need is the ColorSnap 32+. It costs about $\$ 600$. Don't get anything else. For example a very popular card at the moment is the VideoSpigot. This will NOT make acceptable, high quality stills for publication. It's designed to grab a "movie," a rapid sequence of frames, but not a single frame at the best possible resolution. Being able to incorporate "movies" i.e. actual video clips, in the computer versions of our analysis offers very interesting possibilities for the future. However here we are concentrating on getting an image from a videotape that can in incorporated in a paper for publication. Because of inherent limitations of the video medium frame grabs will not be high quality graphics (basically their resolution is limited to $72 \mathrm{DPI}$ ). However, while that might not be acceptable for a graphic artist intending to publish in a magazine, it is quite sufficient for the work we are doing, and offers new ways of thinking about, and analyzing, the organization of talk-in-interaction. 
- Mail-order. You frequently get the best prices, as well as excellent service by ordering from large Mail Order house such as MacConnection. Up to date ads can be found in the current issues of MacWorld and MacUser. Mail-order houses usually deliver the next day with reasonable shipping charges.

- Monitors. For both Frame Grabbing and general work a large two page (e.g. 21") monitor is excellent. I use black and white (actually 256 gray scale). Since everything I print has to go to black and white I don't want to become dependent on color. Moreover black and white monitors are much, much cheaper than color monitors. MacWorld reviewed these monitors in its July 1992 issue and recommended the Raster Ops 19" and the Radius TDP/21.

\section{Frame Grabs in Adobe Photoshop, Basic Procedure}

1) Make the frame grab

2) Change the Mode to Gray Scale (shifts file size from $900 \mathrm{~K}$ to $300 \mathrm{~K}$ and you aren't going to print in color anyway).

3) Use the Video De-interlace filter

4) Use the Unsharp Mask Filter

- Use the Levels control to adjust contrast, etc.

- If you want to change the Image Size first Uncheck the File Size box.

- If you want to embed the image in Word 5 save it as EPS.

- If you want to Publish the image in Word 5 Create Publisher and then Subscribe to it in Word 5 (This takes far less space than embedding but doesn't print as well).

\section{Cable Setup for Frame Grabs}

- If is very helpful, indeed almost necessary, to be able to see the tape you are grabbing from on a monitor as well as your computer screen. With my card the computer screen does not refresh the image often enough to allow me to see the precise place where I want to make the grab.

- Therefore you want the signal from your recorder to go both to your computer and to your monitor.

- Originally I "looped" the signal through the monitor to the computer. However I found that the quality of the images I was getting with frame grabs was degraded. Therefore you want the signal from you VCR to go to two places: The frame grab card in your computer and your monitor.

- To do this

${ }^{\circ}$ Connect the Video Out Jack on the back of your VCR (the one with RCA plugs like you see on your stereo system) to the Frame Grab card. 
- Connect the VHF out pin on the back of your VCR into the VHF input or antenna input of your monitor. Set the monitor to TV and you can view the image on either Channel 3 or Channel 4 (you can usually choose one of these two channels with a switch on your VCR). This pin uses a single coaxial cable for both video and audio which are combined together. It is the kind of cable and connector that cable TV companies use.

\section{Computer}
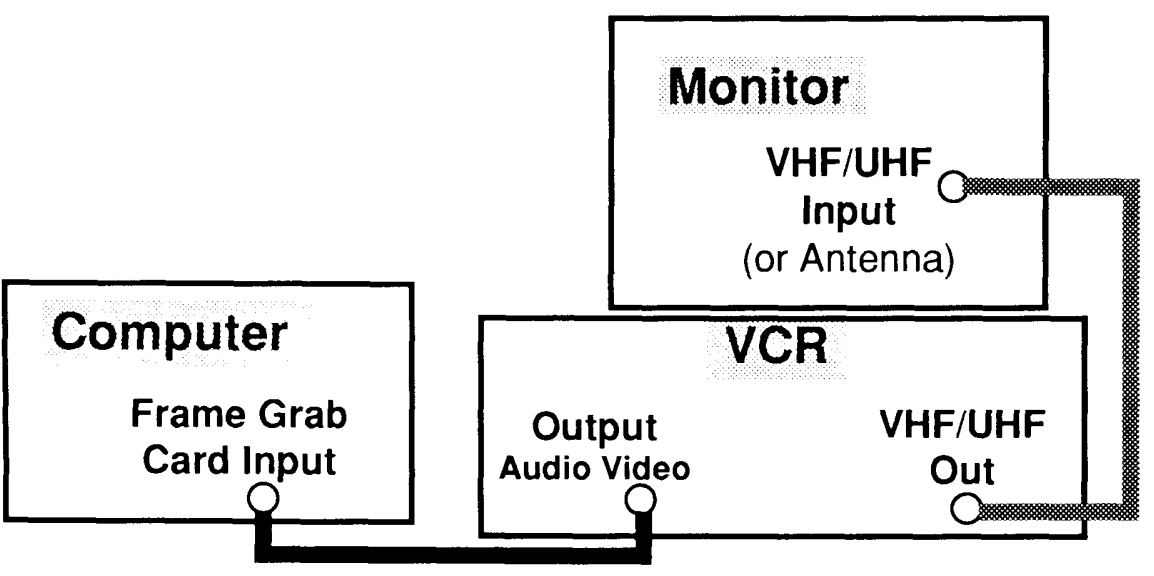

- You get a better signal with the RCA Video Out connections. When you don't want to do frame grabs you can remove the Frame Grab cable from the VCR out jack on the back of your recorder and put the cable from the Video In jack of your monitor back in.

- I use a second method when making Frame Grabs from my Hi-8 camera, but this requires a monitor which has an $\mathrm{S}$ video input. The camera has an $S$ video output jack and cable. I run this to the $S$ video input on the monitor, and hook the Frame Grab Cable into the RCA video out jack on the camera.

\section{Sample Release Form}

This is a release form I've used for some of my taping of scientists. It's offered only as a sample, and no claims whatsoever are made that it will fulfill all legal or human subjects requirements. 


\section{Release for Videotaping}

I (Charles Goodwin) am engaged in a project investigating how the work of science is done. To do this it is essential that I have video and audio tapes of scientists as they actually go about their work and talk with each other. (In previous research I have used videotape to investigate human interaction, with particular attention to the organization of talk; the present project is part of an effort to extend such analysis to larger social institutions). The videotapes that I am recording are for research purposes only. They will not be broadcast or put to any commercial use. They will only be shown to others in the context of professional work.

I have read the above and agree to be taped.

Name:

Address

Signature

Date: 